\title{
Particle Creation by a Black Hole as a Consequence of the Casimir Effect
}

\author{
R. M. Nugayev \\ Kazan State Pedagogical Institute, SU-420021 Kazan, USSR
}

\begin{abstract}
Particle creation by a black hole is investigated in terms of temperature corrections to the Casimir effect. The reduction of the Hawking effect to more familiar effects observed in the laboratory enables us to reveal the mechanism of particle creation. The blackbody nature of the Hawking radiation is due to the interaction of virtual particles with the surface of a "cavity" formed by the Schwarzschild gravitational field potential barrier. These particles are "squeezed out" by the contraction of the potential barrier and appear to an observer at $J^{+}$as the real blackbody ones.
\end{abstract}

In the previous papers [2-4] a programme of reduction of particle creation by a black hole to quantum- field effects in flat space-time was initiated. The programme is based on the fact that the gravitational field of a black hole creates an effective potential barrier penetrable for the high-frequency waves and impenetrable for waves with low-frequency. The barrier is so well-localized near $r=1.5 \mathrm{Rg}$ $\left(R g=+2 G M / c^{2}\right)$ that for a study of wave propagation we can consider the regions quite near the horizon and far away from it as "flat". All the scattering takes place in the small region near $r=1.5 \mathrm{Rg}$. The consideration of the barrier peak $(r=1.5 \mathrm{Rg})$ as a surface of the reflecting sphere permitted to apply to a black hole the results of various Casimir-effect calculations. It appeared [2] that the flow of negative Casimir energy should cause the area of the horizon to shrink at a rate consistent with the energy flux observed at future infinity $J^{+}$. But the model appeared to be too primitive providing only qualitative agreement with Hawking's results [1].

Hence the second stage of the programme had to be carried out [3]. It consisted in creation of a more sophisticated model capable of demonstrating that the two properties of a black hole - the horizon and the potential barrier - together are necessary and sufficient to compel the hole to produce thermal radiation at a temperature that exactly coincides with the result of Hawking. Namely that was done by means of the reduction of the evaporation effect to that of particle creation by (non-uniformly) accelerated mirrors. 
But even the second model remained too simple to mimic some important features of the evaporation process since the vacuum stress-tensor diverged in the reference frame of a freely falling observer as $r \rightarrow 2 M$. The second model pathology is due to the assumption of ideal conductivity which is obviously not the case for a spherical potential barrier of a nonrotating black hole. So, the third stage of the programme was carried out [4] and the finite conductivity of the barrier was taken into account. It enabled one to eliminate the pathology and to reveal simultaneously that the blackbody radiation should be "created" in the whole region $[3 M, \infty]$.

However, even the third model is able to describe the creation domain only, but not the very mechanism of black hole evaporation as well as the origin of why the radiation at $J^{+}$is the blackbody radiation. The cause of it is obvious: the third model ignores the thickness of the potential barrier. In [2-4] the barrier was approximated by a thin shell. However, Fabbri [5] demonstrated that there exists two branches of turning points for a nonrotating black hole potential barrier:

$$
\left.\begin{array}{l}
r_{1}=\frac{2}{\omega}\left[\frac{\ell(\ell+1)}{3}\right]^{1 / 2} \operatorname{Cos} \frac{\eta}{3} \\
r_{2}=\frac{2}{\omega}\left[\frac{\ell(\ell+1)}{3}\right]^{1 / 2} \operatorname{Cos} \frac{\eta \sim 2 \pi}{3}
\end{array}\right\},
$$

where $\eta=\arccos \left\{-3 \omega M[3 / \ell(\ell+1)]^{1 / 2}\right\}$ and arccos denotes the principal value of the inverse trigonometric function, so that $2 M \leqq r_{1} \leqq r_{2}$. For instance, each $(\omega, \ell)$ partial low-frequency wave has two turning points,

and

$$
r_{1}=2 M\left[1+\frac{4 \omega^{2} M^{2}}{\ell(\ell+1)}+O\left(\frac{\omega^{4} M^{4}}{\ell^{4}}\right)\right]
$$

$$
\left.r_{2}=\frac{[\ell(\ell+1)]^{1 / 2}}{\omega}-M\left[1+O\left(\frac{\omega M}{\ell}\right)\right]\right\} \text {, }
$$

where $O(x)$ denotes a quantity of order $x$.

Consequently, for purpose of investigating the interaction of virtual particles with the surface of the potential barrier, the latter should be represented by two conducting concentric spheres. One of the shells is situated near the horizon while the other is far away from it. Each sphere is made of an ideal conductor. The aim of the paper is to give a description of the evaporation mechanism on the basis of this model.

A. Casimir [6] demonstrated that the vacuum fluctuations of the electromagnetic field give rise to an attractive force between conducting parallel plates. When one quantizes the field subject to the appropriate boundary conditions on the plates and calculates the vacuum energy with a wavelength cutoff, one finds that as the separation between the plates changes, the vacuum energy per unit area changes by a finite, cutoff-independent amount. Thus, in spite of the formal divergence of vacuum energy, a change in the configuration of the system causes a finite shift in the energy of the vacuum state. If the vacuum energy of the system for infinite separation is set equal to zero, then the energy of the plates for any finite separation 
is described by the expression

$$
\Delta E=-\pi^{2} \hbar c \mathscr{A} / 720 d^{3} .
$$

Here $\mathscr{A}$ denotes the area of each plate, and $d$ is a finite separation between them.

It should be specially pointed out that the Casimir energy is of pure vacuum origin. No real particles are involved, only virtual ones. But the experiments of Derjagin, Sparnaay, van Silfhout and Tabor and Winterton (see [7] and references cited therein) encourage us to take it seriously.

For the electromagnetic field the stress-tensor of the vacuum between the plates was calculated by De Witt [8]:

$$
\begin{aligned}
\left\langle T^{\mu v}\right\rangle_{\mathrm{vac}}= & T_{(-)}^{\mu v}+T_{(+)}^{\mu v}=\frac{\pi^{2} \hbar c^{2}}{720 d^{4}} \operatorname{diag}(-1,1,1,3) \\
& +\frac{3 \Lambda^{4} \hbar c^{2}}{\pi^{2}} \operatorname{diag}\left(1, \frac{1}{3}, \frac{1}{3}, \frac{1}{3}\right)
\end{aligned}
$$

where $\Lambda$ is the frequency cutoff that cuts off the high-frequency waves. (The expression for the vacuum stress-tensor of the massless scalar field differs from that for the electromagnetic field only by the factor $\frac{1}{2}$.)

The works of Boyer [7,9] offer a method for calculating the vacuum energy of the uncharged sphere made from a physically realizable conductor. Let us approximate a sphere of radius $d$ by two parallel plates of area $\pi d^{2}$ at a distance apart. With the help of (1) and (2) we can obtain

$$
\Delta E=-\pi^{2} \hbar c / 720 d+3 \hbar c \Lambda^{4} d^{3} / \pi
$$

where the second part is a correction for finite conductivity of the plates. The approximation is justified by the exact calculations of Boyer [9] and Davies [10] performed independently. Having computed the vacuum energy of a sphere with ideal conductivity, they demonstrated that $\Delta E$ exactly coincides in magnitude with the cutoff-independent part of (3). Only the sign changes. So, for finite conductivity,

$$
\Delta E=\pi^{3} \hbar c / 720 d-3 \hbar c \Lambda^{4} d^{3} / \pi
$$

All the studies of massless fields in various metallic cavities, initiated by Boyer and reconsidered by several groups, have focused on the integrated total energy. Olaussen and Ravndal [11] seem to be one of the first to undertake the more detailed and laborious analysis of local densities for spherical cases.

The energy density

$$
\langle\Omega \mid: u(r): \Omega\rangle \cong-\frac{1}{d^{4}}\left\{\frac{4}{15 \pi^{2} \delta^{3}}-\frac{2}{35 \pi^{2} \delta^{2}}+0.005\left[1+\left(\frac{r}{d}\right)^{2}\right]\right\},
$$

where

$$
\delta=\left[1-\left(\frac{r}{d}\right)^{2}\right]
$$


Near the surface of the sphere where $r \rightarrow d$,

$$
\langle\Omega \mid: u(r): \Omega\rangle \cong \frac{1}{\pi^{2} d^{4}}\left(\frac{-1}{30 \varepsilon^{3}}\right), \quad \text { where } \quad \varepsilon=1-\frac{r}{d} .
$$

So, the (normal ordered) energy density is found to be negative everywhere inside the cavity. In that respect a spherical cavity is not qualitatively different from the parallel plate or box geometries. The unexpected result, first found by Boyer, that the total Casimir effect for a spherical shell actually leads to an increase in energy only occurs because the energy density outside the shell is positive enough to overcompensate the negative energy inside.

The stresses induced in the Minkowski vacuum by an infinite plane conductor that is uniformly accelerated normal to itself were investigated by Candelas and Deutsch (see [16]). The solution of the boundary problem was facilitated by introduction of accelerated (Rindler) coordinates $\xi$ and $\tau$,

$$
t=\xi \sinh \tau, \quad x=\xi \cosh \tau, \quad d s^{2}=\xi^{2} d \tau^{2}+d \xi^{2}+d y^{2}+d z^{2} .
$$

In this system the curves $\xi=$ const, $y=$ const, $z=$ const are worldlines of constant proper acceleration $\xi^{-1}$. The surface $\xi=b=$ const represents the trajectory of the barrier.

The regularized vacuum expectation value $\left\langle T_{v}^{\mu}\right\rangle=\left\langle 0\left|T_{v}^{\mu}\right| 0\right\rangle$ of the stress energy tensor far from the conductor $(\xi / b \rightarrow \infty)$ for a scalar field was found to be

$$
\left\langle T_{v}^{\mu}\right\rangle \sim-\frac{1}{2 \pi \xi^{4}} \int_{0}^{\infty} \frac{d \omega \omega^{3}}{e^{2 \pi \omega}-1} \operatorname{diag}\left(-1, \frac{1}{3}, \frac{1}{3}, \frac{1}{3}\right) .
$$

Thus $\left\langle T_{v}^{\mu}\right\rangle$ is reduced below zero by an amount corresponding to black body radiation at a temperature

$$
T=(2 \pi \xi)^{-1}
$$

This asymptotic form is independent of the acceleration of the barrier in the sense that it depends only on the acceleration of the local Killing trajectory.

Or, equivalently, far from the conductor

$$
\begin{aligned}
\left\langle T_{\mathscr{D} v}^{\mu}\right\rangle= & {\left[-\left(480 \pi^{2} \xi^{4}\right)^{-1}-\left(144 \xi^{4} \ln ^{3} \xi / b\right)^{-1}\right] \operatorname{diag}\left(-1, \frac{1}{3}, \frac{1}{3}, \frac{1}{3}\right) } \\
& +O\left[\left(\xi^{4} \ln ^{4} \xi / b\right)^{-1}\right], \\
\left\langle T_{\mathcal{V}_{\nu}}^{\mu}\right\rangle= & {\left[-\left(480 \pi^{2} \xi^{4}\right)^{-1}+\left(288 \xi^{4} \ln ^{3} \xi / b\right)^{-1}\right] \operatorname{diag}\left(-1, \frac{1}{3}, \frac{1}{3}, \frac{1}{3}\right) } \\
& +O\left[\left(\xi^{4} \ln ^{4} \xi / b\right)^{-1}\right],
\end{aligned}
$$

where $\left\langle T_{\mathscr{D} v}^{\mu}\right\rangle$ and $\left\langle T_{\mathscr{N} v}^{\mu}\right\rangle$ denote the values of $\left\langle T_{v}^{\mu}\right\rangle$ for the Dirichlet and Neumann cases respectively.

The results belonging to temperature corrections to the Casimir effect (M. Fiertz, J. Mehra, R. Hargreave, Brown and Maclay et al.) were generalized by Tadaki and Takagi [12] for two parallel infinite plane boundaries in the fourdimensional Minkowski spacetime. This system has two special directions $(t, z)$ because of the presence of the boundaries and the heat bath. According to the 
symmetry of the system, the conservation law and the tracelessness, $\left\langle T_{\mu \nu}\right\rangle$ has the following form;

$$
\begin{aligned}
\left\langle T_{\mu \nu}\right\rangle= & \mathscr{A} \operatorname{diag}(-1,1,1,-3)+\mathscr{B} \operatorname{diag}(3,1,1,1)+\mathscr{C} \operatorname{diag}(1,0,0,1) \\
& +F \operatorname{diag}(2,1,1,0)
\end{aligned}
$$

It is remarkable that $\left\langle T_{33}\right\rangle \equiv\left\langle T_{z z}\right\rangle$ is uniform though the other diagonal components of $\left\langle T_{\mu \nu}\right\rangle$ are possibly $z$-dependent. The first term in (7) represents the zerotemperature term and the second the Stephan-Boltzmann term. For a conformally coupled massless scalar field

$$
\begin{gathered}
\mathscr{A}=\frac{\pi^{2} \hbar c}{1440 d^{4}}, \quad \mathscr{B}=\frac{\pi^{2} k^{4} T^{4}}{90 \hbar^{3} c^{3}}, \quad \mathscr{C}=\frac{\hbar c}{2 d^{4}}\left\{3 f(T)-\frac{k d}{\hbar c} T f^{\prime}(T)\right\}, \\
F(z)=\left\{-\frac{1}{2} f(T)+\frac{1}{6} \frac{k d}{\hbar c} T g^{\prime}(T, z)\right\} \frac{\hbar c}{d^{4}},
\end{gathered}
$$

where

$$
\begin{gathered}
f(T)=-\frac{1}{4 \pi^{2}} \sum_{n=1}^{\infty} \sum_{m=1}^{\infty} \frac{(2 k d T / \hbar c)^{4}}{\left[m^{2}+4 k^{2}(k d T / \hbar c)^{2}\right]^{2}}, \quad f^{\prime}(T)=\frac{d f(T)}{d T}, \\
g(T, z)=-\frac{1}{8 \pi^{2}} \sum_{n=-\infty}^{\infty} \sum_{m=1}^{\infty} \frac{(2 d k T / \hbar c)^{4}}{\left[m^{2}+4(z / d+n)^{2}(k d T / \hbar c)^{2}\right]^{2}}, \quad g^{\prime}(T, z)=\frac{\partial g}{\partial T} .
\end{gathered}
$$

In the low-temperature limit $(T d \ll 1),\left\langle T_{\mu \nu}\right\rangle$ has a sinusoidal $z$-dependence:

$$
\begin{gathered}
\mathscr{A}=\frac{\pi^{2} \hbar c}{1440 d^{4}}, \quad \mathscr{B}=0, \quad \mathscr{C}=\frac{\pi k T}{2 d^{3}} e^{-\pi \hbar c / k d T}+O\left(T^{2} e^{-2 \pi / T}\right), \\
F(z)=\frac{k T}{d^{3}} e^{-\pi \hbar c / k d T}\left\{-\frac{\pi}{6} \operatorname{Cos} 2 \pi z / d+\frac{k d T}{2 \hbar c}\left(1+\frac{k d T}{\hbar c \pi}\right)\right. \\
\times(1-\operatorname{Cos} 2 \pi z / d)\}+O\left(T^{2} e^{-2 \pi / T}\right) .
\end{gathered}
$$

Here the Stephan-Boltzmann term is cancelled out. The temperature correction is exponentially small, because the basis modes have an energy gap.

In the high temperature limit $(T d \gg 1),\left\langle T_{\mu \nu}\right\rangle$ is dominated by the StephanBoltzmann value everywhere not close to the boundary. The behaviour near the boundary may be seen by considering the single boundary problem. In the limit $d \rightarrow \infty$ the result is

$$
\begin{gathered}
\mathscr{A}=0, \quad \mathscr{B}=\frac{\pi^{2} k^{4} T^{4}}{90 \hbar^{3} c^{3}}, \quad \mathscr{C}=0, \\
F(z)=\frac{\pi^{2} T^{4} k^{4}}{12 \hbar^{3} c^{3}}\left\{2 Z^{-1} \operatorname{Coth} Z \operatorname{Cosec}^{2} Z-Z^{-2} \operatorname{Cosec}^{2} Z-Z^{-3} \operatorname{Coth} Z\right\},
\end{gathered}
$$

where

$$
Z=2 \pi T z k / \hbar c
$$


The thermal average deviates off the Stephan-Boltzmann value near the boundary $(Z \ll 1)$ due to the $T^{4}$ term of $F(z)$ :

$$
F(z)=-\frac{\pi^{2} T^{4} k^{4}}{90 \hbar^{3} c^{3}} \cdot \frac{4}{3}\left\{1-\frac{2}{7} Z^{2}+\frac{2}{35} Z^{4}+O\left(Z^{6}\right)\right\} .
$$

This expansion coincides with the result obtained in [13]. The calculations for the electromagnetic field are almost the same.

And, finally, Levin et al. [14] obtained, with the help of generalized Kirchhoff's $1 \mathrm{aw}{ }^{1}$, the expressions for spectral and complete Poynting vector of the fluctuating electromagnetic field in a vacuum cavity formed by infinite flat parallel conductors $\left(\varepsilon_{1}, \mu_{1}\right)$ and $\left(\varepsilon_{2}, \mu_{2}\right)$ with temperatures $T_{1}$ and $T_{2}\left(T_{1}>T_{2}\right)$. The Poynting vector

where

$$
P=\int_{0}^{\infty} p(\omega) d \omega=\frac{1}{\pi^{2}} \int_{0}^{\infty}\left(\Pi_{1}-\Pi_{2}\right) M d \omega,
$$

$$
\Pi_{i}=\frac{\hbar \omega}{\exp \left(\hbar \omega / K T_{i}\right)-1}, \quad i=1,2 ; \quad k=\frac{\omega}{c} .
$$

In vacuum $\left(\varepsilon_{1}=\varepsilon_{2}=\mu_{1}=\mu_{2}=n=1\right)$ for infinite separation $(d \rightarrow \infty)$ one gets

$$
M(\infty)=\frac{k^{2}}{8} ;
$$

under

$$
\begin{aligned}
& d=0, \quad M(0)=\frac{k^{2}}{4}, \\
& P(0)=\sigma_{S B}\left(T_{1}^{4}-T_{2}^{4}\right) .
\end{aligned}
$$

Thus, though each conductor is in equilibrium with radiation but each at different temperatures, the whole system is in nonequilibrium state. Under these conditions a flow of the fluctuating electromagnetic field from $T_{1}$ to $T_{2}\left(T_{1}>T_{2}\right)$ dominates inside the cavity over the flow from $T_{2}$ to $T_{1}$.

B. Consider a particle which is at rest in the gravitational field of a Schwarzschild black hole. Its four-velocity is

$$
u^{\alpha} \equiv \frac{d x^{\alpha}}{d \tau}=\left(\left(1-\frac{2 M}{r}\right)^{-1 / 2}, 0,0,0\right)
$$

The proper acceleration of the particle is

$$
a^{\alpha} \equiv \frac{\mathscr{D} u^{\alpha}}{d \tau}=\frac{d u^{\alpha}}{d \tau}+\Gamma_{\beta}^{\alpha} u^{\beta} u^{\gamma}=\Gamma_{t t}^{\alpha} u^{t} u^{t}
$$

\footnotetext{
${ }^{1}$ The authors of [14] especially point out that the generalized Kirchhoff's law contains an expression for the oscillator's average energy $\theta(\omega, T)$. Nevertheless, zero oscillations does not give any impact into the energy flow and are thrown away here: $\Pi_{1}=\theta(\omega, T)-\hbar \omega / 2$. But, of course, the energy of the equilibrium fluctuating electromagnetic field is $E=\sum_{\alpha} \frac{\hbar \omega_{\alpha}}{2}$ $+\sum_{\alpha} \hbar \omega_{\alpha} / \exp \left(\hbar \omega_{\alpha} / K T\right)^{-1}$, where $\omega_{\alpha}$ are the eigenfrequencies, depending on $d$
} 
$(\alpha, \beta, \gamma=t, r, \theta, \varphi)$. The only nonvanishing component of $\Gamma_{t t}^{\alpha}$ is $\Gamma_{t t}^{r}=\frac{M}{r^{2}}\left(1-\frac{2 M}{r}\right)$. Hence $a^{\alpha}=\left(0, \frac{M}{r^{2}}, 0,0\right)$,

$$
|a|=\left(g_{\alpha \beta} a^{\alpha} a^{\beta}\right)^{1 / 2}=\left(1-\frac{2 M}{r}\right)^{-1 / 2} \frac{M}{r^{2}} .
$$

A stationary distant observer will measure

$$
\begin{gathered}
b^{\alpha}=\frac{\mathscr{D} u^{\alpha}}{d \tau} \frac{d \tau}{d t}=a^{\alpha}\left(1-\frac{2 M}{r}\right)^{1 / 2}, \\
|b| \equiv\left(g_{\alpha \beta} b^{\alpha} b^{\beta}\right)^{1 / 2}=\frac{M}{r^{2}} .
\end{gathered}
$$

Consequently, the peak of the potential barrier (localized in the vicinity of $r=3 M)$ has a nonzero proper acceleration $\cong(3 \sqrt{3} M)^{-1}$.

C. According to Fabbri [5], who studied the scattering and absorption of electromagnetic waves by a nonrotating black hole, when the frequency $\omega$ of radiation is smaller than the critical frequency $\omega_{c}$ given by

$$
\omega_{c}=(2 / 27)^{1 / 2} M^{-1}
$$

the turning points exist for all partial waves, that is, for all values of $\ell$. When $\omega>\omega_{c}$, the turning points exist only for high- $\ell$ waves; more precisely, they exist if $\ell$ is greater than the critical parameter $\ell_{c}$ given by

$$
\ell_{c}\left(\ell_{c}+1\right)=27 \omega^{2} M^{2} \text {. }
$$

At high frequencies $\left(\omega \gg \omega_{c}\right)$, for $\ell \ll \ell_{c}$, the waves pass above the potential barrier completely unaffected. When $\ell$ is slightly greater than $\ell_{c}$ the turning points are approximately given by

$$
r_{1,2}=3 M\left\{1 \mp \frac{1}{\sqrt{3}}\left[1-\frac{27 \omega^{2} M^{2}}{\ell(\ell+1)}\right]^{1 / 2}\right\} .
$$

In the case $\ell<\ell_{c}$ the zeros of the wave number are given by

$$
\bar{r}_{1,2}=3 M\left\{1 \mp \frac{i}{\sqrt{3}}\left[1-\frac{\ell(\ell+1)}{27 \omega^{2} M^{2}}\right]^{1 / 2}\right\} .
$$

So, for $\omega>\omega_{c}$ the transmission coefficient of the barrier is

$$
\left.\begin{array}{lll}
T_{\ell}=0 & \text { at } & \ell>\ell_{c} \\
T_{\ell}=1 & \text { at } & \ell<\ell_{c}
\end{array}\right\}
$$

For $\omega \ll \omega_{c}$ real turning points exist for all partial waves [Eq. (i)]:

$$
T_{\ell}=4\left[\frac{(\ell+1) !(\ell-1) !}{(2 \ell) !(2 \ell+1) ! !}\right]^{2}(2 \omega M)^{2 \ell+2} .
$$


That is why they are only the low-frequency waves that can successfully escape from the region formed by the Casimir plates with reflecting picture nicely mimicked by the expressions (i). This conclusion is also justified by the calculations of Sanchez [15]: the reflecting properties of the potential barrier provide that Hawking emission is only significant in the frequency range $0 \leqq \omega<\frac{1}{M}$. Consequently, the potential barrier of a nonrotating black hole should be approximated by two concentric shells with the first in the vicinity of the horizon $\left(r=r_{1}=2 M+4 \omega^{2} M^{3} / \ell(\ell+1) \rightarrow 2 M, \omega \rightarrow 0\right)$ and the second far away from it $\left(r=r_{2} \cong \sqrt{\ell(\ell+1)} / \omega \rightarrow \infty, \omega \rightarrow 0\right)$. Success of the approximation of the Casimir sphere by two parallel plates - Eqs. (1)-(4) - permits us to replace each spherical conductor by two plane conductors.

Consider an observer resting on the surface of one such conductor $\left(r=r_{0}\right)$ in the gravitational field of a Schwarzschild black hole. According to the Principle of Equivalence, he is equivalent to an observer accelerated in Minkowski spacetime with proper acceleration $b^{-1}=\left(1-\frac{2 M}{r_{0}}\right)^{-1 / 2} \frac{M}{r_{0}^{2}}$. However, as it is well-known (see, for example, [16], and the references cited therein), an observer that is accelerated in Minkowski spacetime with a proper acceleration $b^{-1}$, finds himself in a thermal bath with temperature $T=b^{-1} / 2 \pi c k$. An observer who is accelerated with the surface of the wall, should find the thermal radiation being in equilibrium with the wall at the same temperature. Hence, an observer resting on the surface of a conductor in the gravitational field of a Schwarzschild black hole would discover thermal radiation in equilibrium with a conductor at a temperature

$$
T=\frac{M}{r_{0}^{2}\left(1-\frac{2 M}{r_{0}}\right)^{1 / 2} 2 \pi c k} \text {. }
$$

Consequently the interaction of the radiation with the surface of the potential barrier can be described in terms of temperature corrections to the Casimir effect.

Firstly, the temperature $T_{1}$ of a conductor in the vicinity of the horizon is considerably higher than that of a conductor far aray from it. So, though each conductor is in equilibrium with radiation, the whole system is in a nonequilibrium state $\left(T_{1}>T_{2}\right)$, and a flow of the fluctuating scalar (or electromagnetic) field establishes itself in the region $\left[r_{1}, r_{2}\right]$. The flow is directed from the horizon to spatial infinity.

An observer who sits at rest $\left(r=r_{0}\right)$ near the horizon will discover a flow of thermal radiation with a temperature $T_{1}=\frac{1}{2 \pi} \frac{M}{r_{0}^{2}}\left(1-\frac{2 M}{r_{0}}\right)^{-1 / 2} \cdot$ A distant stationary observer at future infinity $\mathscr{I}^{+}$will find that the temperature of radiation in the vicinity of the horizon is $T=\frac{1}{2 \pi} \frac{M}{r_{0}^{2}}$. Indeed, the gravitational blue shift of the photon (ratio of observed energy $\hbar \omega_{0}$ to emitted at $\mathscr{I}^{+}$energy $\hbar \omega$ ) is $\omega_{0} / \omega=\left(g_{\omega}\right)^{-1 / 2}\left(1-\frac{2 M}{r_{0}}\right)^{-1 / 2}$. But $\frac{\omega}{T}=$ const along the light ray (see [17]). That is why $T_{1}=T\left(1-\frac{2 M}{r_{0}}\right)^{-1 / 2}$. 
According to (15), $\frac{M}{r_{0}^{2}}$ is the magnitude of the acceleration (measured by an observer at $\mathscr{I}^{+}$) of a particle at rest in the gravitational field of a Schwarzschild black hole. It tends (see [18]) to the so-called "surface gravity" $\kappa$ when the particle is infinitesimally close to the event horizon. For a Schwarzschild black hole $\kappa=(4 M)^{-1}(c=G=1)$. So, the temperature of the radiation near the horizon is $T_{1}=\kappa / 2 \pi$ according to an observer at $\mathscr{I}^{+}$. Since the temperature $T_{2}$ of an observer far away from the horizon is negligible, the Poynting vector [see Eqs. (11) and (12)] is

$$
\begin{aligned}
P & =\int_{0}^{\infty} p(\omega) d \omega=\frac{1}{\pi^{2}} \int_{0}^{\infty}\left(\Pi_{1}-\Pi_{2}\right) M d \omega \\
& =\frac{1}{\pi^{2}} \int_{0}^{\infty} \frac{\hbar \omega M(\infty) d \omega}{\exp \left[\hbar \omega / K T_{1}\right]-1}=\frac{1}{\pi^{2}} \int_{0}^{\infty} \frac{\hbar \omega^{3} d \omega}{\exp \left[\hbar \omega / K T_{1}\right]-1} \\
& =\frac{\hbar}{\pi^{2} c^{2}} \frac{1}{\left(1-\frac{2 M}{r}\right)^{2}} \int_{0}^{\infty} \frac{\omega_{0}^{3} d \omega_{0}}{\exp \left[\hbar \omega_{0} 2 \pi / K \kappa\right]-1} .
\end{aligned}
$$

Equation (23) exactly coincides with the results of the various studies of Hawking radiation made on the basis of usual quantum field theory in curved spacetimes. It should be pointed out that zero oscillations does not give any direct impact into the energy flow (23). But, of course, they influence it through the expression for the energy of the equilibrium fluctuating electromagnetic field $E=\sum_{\alpha}\left(\hbar \omega_{\alpha} / 2+\hbar \omega_{\alpha} / \exp \left[\hbar \omega_{\alpha} / K T\right]-1\right)$ when the eigenfrequencies $\omega_{\alpha}$ depend on $d$.

Secondly, to give a more complete description of the vacuum stress-tensor between the conductors and in the whole $[2 M, \infty]$ region we can apply (7.a)-(7.b) with $d$ being the "distance" in the accelerated (or Rindler - see [17] for details) frame of reference: $d=\xi=\left(1-\frac{2 M}{r}\right)^{1 / 2} \frac{r^{2}}{M}$. But the fact that the temperature $T=T(r)$ varies from one point to another hampers the direct utilization of the temperaturecorrection results. Hence we shall calculate the $\left\langle T_{v}^{\mu}\right\rangle_{\text {vac. }}$ in the vicinities of $r_{1}$ and $r_{2}$ first. In these regions the variations of $T$ with distance are small in comparison with

those in the domain between the conductors.
The proper acceleration of the $r_{2}$ barrier is $b_{2}^{-1}=\frac{M}{\Delta_{2}^{2}}\left(1-\frac{2 M}{\Delta_{2}}\right)^{-1 / 2}$, where $\Delta_{2}=\left(\frac{[\ell(\ell+1)]^{-1 / 2}}{\omega}-M\right) \rightarrow \infty$ if $\omega \rightarrow 0$. The spherical conductor that is far from the horizon can be represented by two plane conductors with equal temperatures $\frac{b_{2}^{-1}}{2 \pi}$ and accelerations $b_{2}^{-1}$. To describe the region $\left[r_{2}, \infty\right]$ the $d \rightarrow \infty$ limit of Eq. (19) should be involved: $(T d) \gg 1$, and \langle\rangle is dominated by the Stephan-Boltzmann values all over the space,

$$
\mathscr{A}=0, \quad \mathscr{C}=0, \quad \mathscr{B}=\frac{\pi^{2} T^{4}}{90}=\frac{M^{4}}{1440 \pi^{2}\left(1-\frac{2 M}{r}\right)^{2}} .
$$


To describe the situation near the other side of the $r_{2}$ barrier, it should be noted that the spherical conductor can be exchanged with a pair of flat plates that rest in the Schwarzschild gravitational field. So, taking into account Eq. (2), we obtain:

$$
\begin{aligned}
\left\langle T^{\mu v}\right\rangle_{\text {vac. }} & =\frac{\pi^{2}}{1440 d^{4}} \operatorname{diag}(-1,1,1,3) \\
& =\frac{\pi^{2} M^{4}}{1440 r^{8}\left(1-\frac{2 M}{r}\right)^{2}} \operatorname{diag}(-1,1,1,3) .
\end{aligned}
$$

Equations (25a)-(25b) are in good qualitative agreement with Frolov's exact calculations [20] obtained by the usual quantum-field methods for the Boulware vacuum:

$$
\begin{aligned}
\left\langle T_{\mu}^{v}\right\rangle & =\frac{M^{2}}{1440 \pi^{2} r^{6}}\left\{\frac{(2-1,5 x)^{2}}{(1-x)^{2}}\left(-\delta_{\mu}^{v}+4 \delta_{0}^{v} \delta_{\mu}^{0}\right)+6\left(3 \delta_{0}^{v} \delta_{\mu}^{0}+\delta_{1}^{v} \delta_{\mu}^{1}\right)\right\}, \\
x & \equiv \frac{2 M}{r} .
\end{aligned}
$$

The Boulware vacuum $|B\rangle$ is defined by requiring normal modes to be positive frequency with respect to the Killing vector $\frac{\partial}{\partial t}$ with respect to which the exterior region is static. $|B\rangle$ is relevant [16] to the region exterior to a massive body that is only just outside its Schwarzschild radius. The Boulware vacuum corresponds to our familiar concept of the empty state at large radii, but is pathological at the horizon since it diverges in the reference frame of a freely falling observer. In the region near the "nonaccelerated" side of the $r_{2}$ conductor Eq. (25b) corresponds to the absence from the vacuum of blackbody radiation with temperature $T_{2}=b_{2}^{-1} / 2 \pi$. It means that if thermal radiation was added, the resulting state would be indistinguishable, near the $r_{2}$, from the usual Minkowski vacuum. $\left\langle T^{\mu v}\right\rangle_{\text {vac. }}$ in Eq. (25a) is positive, that corresponds to the presence in the $\left[r_{2}, \infty\right]$ region (near the "accelerated" side of the $r_{2}$ conductor) of the positive virtual radiation.

When the barrier is made from the real conductor that conducts well only at high frequencies, the Eq. (25) should be modified to include the correction term [4]:

$$
\begin{aligned}
\left\langle T^{\mu v}\right\rangle_{\text {vac. }} & =T_{1}+T_{2}=T_{1}+\frac{3 \Lambda^{4}}{2 \pi^{2}} \operatorname{diag}\left(1, \frac{1}{3}, \frac{1}{3}, \frac{1}{3}\right) \\
& =T_{1}+\frac{3 \omega_{c}^{4}}{2 \pi^{2}\left(1-\frac{2 M}{r}\right)^{2}} \operatorname{diag}\left(1, \frac{1}{3}, \frac{1}{3}, \frac{1}{3}\right)
\end{aligned}
$$

where $\omega_{c}=\sqrt{\frac{2}{27}} \frac{1}{M}$ is the cutoff frequency for the absorption of massless waves by a nonrotating black hole. The cutoff dependent part of (27) at infinity corresponds to that of an ordinary photon gas. 
The proper acceleration of the $r_{1}$ barrier is

$$
b_{1}^{-1}=\frac{M}{\left(2 M+\Delta_{1}\right)^{2}}\left(1-\frac{2 M}{2 M+\Delta_{1}}\right)^{-1 / 2}, \text { where } \Delta_{1}=\frac{8 M^{3} \omega^{2}}{\ell(\ell+1)} .
$$

If we exchange the $r_{1}$ conductor with two plates at the distance $2 r_{1}$ apart, we can apply Eq. (2) to describe the situation in the $\left[2 M, r_{1}\right]$ region:

$$
\begin{aligned}
\left\langle T^{\mu v}\right\rangle_{\text {vac. }}= & \frac{\pi^{2} M^{4}}{1440 r^{8}\left(1-\frac{2 M}{r}\right)^{2}} \operatorname{diag}(-1,1,1,3) \\
& +\frac{3 \omega_{c}^{4}}{2 \pi^{2}\left(1-\frac{2 M}{r}\right)^{2}} \operatorname{diag}\left(1, \frac{1}{3}, \frac{1}{3}, \frac{1}{3}\right) .
\end{aligned}
$$

Or, equivalently, to obtain the first part of Eq. (28) we can use the $(T d) \ll 1$ limit of Eq. (8) with $d \cong 4 M\left(1-\frac{2 M}{r}\right)^{1 / 2}$ :

$$
\begin{aligned}
& \mathscr{B}=0, \quad \mathscr{C}=0, \quad \mathscr{A}=\frac{\pi^{2}}{1440 d^{4}} \cong \frac{\pi^{2}}{1440(4 M)^{4}\left(1-\frac{2 M}{r}\right)^{2}} ; \\
&\left\langle T_{00}\right\rangle \cong-\frac{\pi^{6}}{90(8 \pi M)^{4}\left(1-\frac{2 M}{r}\right)^{2}} .
\end{aligned}
$$

Equations (28a) - (28b) are justified by the application for [2M, $\left.r_{1}\right]$ of a black hole of Eq. (5b) valid near the surface of an ideal spherical conductor:

where

$$
\langle\Omega|: u(r):| \Omega\rangle \cong-\frac{1}{\pi^{2} d^{4} 30 \varepsilon^{3}} \cong-\frac{M^{4}}{30 \pi^{2} r^{3}\left(1-\frac{2 M}{r}\right)^{2}} \frac{1}{\varepsilon^{3}},
$$

$$
\varepsilon=1-\frac{r}{2 M}\left(1-\frac{2 M}{r}\right)^{1 / 2} .
$$

Again the equations obtained are in good agreement with Frolov's Eq. (26) for Boulware vacuum. The $|B\rangle$ vacuum in $\left[2 M, r_{1}\right]$ is depressed below zero by an amount corresponding to the absence from the vacuum of blackbody radiation at a temperature $T=\frac{1}{8 \pi M\left(1-\frac{2 M}{r}\right)}$. It is this pure virtual Casimir negative energy that enables the black hole to contract with nonuniform acceleration.

The second part of Eq. (28a) enables one to eliminate the pathology of $|B\rangle$ on the horizon.

To describe the situation near the other side of $r_{1}$, in the direction of acceleration, we can again apply the $(T d) \gg 1$ limit of Eq. (19) with $d \rightarrow \infty$. Again 
$\left\langle T_{\mu \nu}\right\rangle$ is dominated by the Stephan-Boltzmann value all over the conductor:

$$
\mathscr{A}=0, \quad \mathscr{C}=0, \quad \mathscr{B}=+\frac{\pi^{2} T^{4}}{90}=\frac{\pi^{2}}{90(8 \pi M)^{4}\left(1-\frac{2 M}{r}\right)^{2}} .
$$

The expression (25b) for the "unaccelerated" side of the barrier can be obtained in the way that clearly points out its physical significance. If we exchange the $r_{2}$ conductor by two plates at the distance $\Delta_{2}\left(1-\frac{2 M}{r}\right)^{1 / 2}$ apart, the $(T d) \ll 1$ limit of Eq. (8) can be involved to describe the situation in the vicinity of $r_{2}$ :

$$
\begin{gathered}
\mathscr{B}=0, \quad \mathscr{C}=0, \quad \mathscr{A}=\frac{\pi^{2}}{1440 d^{4}} \cong \frac{\pi^{2}}{1440\left(1-\frac{2 M}{\Delta_{2}}\right)^{2} \Delta_{2}^{2}} ; \\
\left\langle T_{00}\right\rangle=-\frac{\pi^{2}}{1440\left(1-\frac{2 M}{\Delta_{2}}\right) \Delta_{2}^{2}} .
\end{gathered}
$$

Of course the result is too rough, but it helps to reveal an important detail of the radiation picture between the $r_{1}$ and $r_{2}$ conductors: any observer in the $\left[r_{1}, r_{2}\right]$ region sees two flows of blackbody radiation crossing each other. The dominating flow with $T_{1}=\frac{1}{8 \pi M}$ comes from the $r_{1}$ conductor; and the $r_{2}$ conductor implies the second one, of negative energy. It comes from the surface of $r_{2}$ and corresponds to the absence from the vacuum of blackbody radiation with $T_{2}=\left(2 \pi b_{2}\right)^{-1}$, according to an observer at $\mathscr{I}^{+}$. An observer at $r=r_{0}$ in $\left[r_{1}, r_{2}\right]$ sees

$$
T_{1}=\frac{1}{8 \pi M\left(1-\frac{2 M}{r_{0}}\right)^{1 / 2}}, \quad T_{2}=\frac{1}{2 \pi \xi}=\frac{M}{2 \pi r_{0}^{2}\left(1-\frac{2 M}{r_{0}}\right)^{1 / 2}},
$$

since Eq. (6a)-(6b) guarantee that the asymptotic forms of $\left\langle T^{\mu v}\right\rangle_{\text {vac. }}$ are independent of the acceleration $b_{2}^{-1}$ of the barrier in the sense that they depend only on the acceleration $\xi^{-1}$ of the local Killing trajectory. The resulting flow

$$
\left\langle T^{\mu \nu}\right\rangle=\frac{\pi^{2}}{90}\left[T_{1}^{4}-T_{3}^{4}\right]=\frac{\pi^{2}\left(1-x^{8}\right)}{90(8 \pi M)^{4}(1-x)^{2}}, \quad x \equiv \frac{2 M}{r}
$$

in complete agreement with Eq. (13) for $d=0$.

Equation (31) is also in good qualitative agreement with Page's [21] formulae obtained for the Hartle-Hawking vacuum:

$$
\left\langle T_{\mu}^{v}\right\rangle_{H}=\frac{\pi^{2}}{90(8 \pi M)^{4}}\left\{\frac{\left[1-(4-3 x)^{2} x^{6}\right]\left[\delta_{\mu}^{v}-4 \delta_{0}^{v} \delta_{\mu}^{0}\right]}{(1-x)^{2}}+24 x^{6}\left(3 \delta_{0}^{v} \delta_{\mu}^{0}+\delta_{1}^{v} \delta_{\mu}^{1}\right\} .\right.
$$

The Hartle-Hawking vacuum $|H\rangle$ is defined by taking incoming modes to be positive frequency with respect to the null coordinate on the future horizon, and outgoing modes to be positive frequency with respect to the null coordinate on the 
past horizon. $|H\rangle$ corresponds to a black hole in equilibrium with an infinite sea of blackbody radiation. This equilibrium is unstable (see [16]) since the temperature of the hole varies inversely with its mass. So if, by virtue of a fluctuation, the black hole were to absorb more radiation than it emitted, its mass would increase and hence its temperature would fall. It would absorb more radiation, cool further, etc. On the other hand, if the black hole were initially to emit more radiation than it absorbed then its temperature would rise. It would radiate more rapidly. So in either case all the system as a whole tends to evolve away from equilibrium. However, the stability of the equilibrium can be restored by enclosing the black hole in a suitably small box [22] as we had done already.

Thus, all the thermal radiation is "born" in all the "region" $\left[r_{1}, r_{2}\right]$ between the conductors. Its blackbody spectrum owes to the interaction of scalar, electromagnetic, etc. fluctuations with the conductor surfaces. The dominating flow is directed from $r_{1}$ to $r_{2}\left(T_{1}>T_{2}\right)$. The particles between the conductors still are virtual ones. And they would remain virtual if this was the case for real black holes. Yet it is only the scattering aspect of the Schwarzschild gravitational field that entered our ideal model. The exchange of the nonrotating black hole potential barrier with two ideal conductors is merely an approximation. The real potential barrier of a black hole forms a "bell" that lasts continuously from zero magnitude at the horizon up to zero at spatial infinity passing through the maximum at $r=3 \mathrm{M}$. The reflecting properties (i), (17)-(21) insure that the barrier behaves as a real, and not as an ideal, conductor which conducts well at low frequencies; but as the frequencies increase, its conductivity diminishes. So, the Hawking radiation is "born" inside the "bell" formed by a potential barrier of a nonrotating black hole in all the region $[2 M, \infty]$. Its blackbody spectrum owes to the interaction of zero-rest-mass field fluctuations with the surface of the "bell". The flow is directed from the $[2 M, 3 M]$ region to the $[3 M, \infty]$ tail of the potential barrier. The particles between the walls of the bell are virtual ones. But they can become real after passing through the $[3 M, \infty]$ tail, appearing to an observer at future infinity $\mathscr{I}^{+}$as real ones, created by the accelerated tail of the potential barrier.

The comparison of our reaults with those obtained by usual and more laborious methods of quantum-field theory in curved spacetimes gives one a confidence that the proposed picture does not differ significantly from the "real" one. For instance, the situation at $r=3 M$ with no flux corresponds to unstable photons that circle around the horizon of a Schwarzschild black hole.

Acknowledgements. It is a pleasure to thank R. Fabbri, S. A. Fulling, N. Sanchez, and J. W. York for helpful comments and for sending me reprints of their papers. The author is grateful to E. Permyakov, D. Galtzov, and Yu. Gnat'ev for valuable discussions.

\section{References}

1. Hawking, S.W.: Particle creation by black holes. Commun. Math. Phys. 43, 199 (1975)

2. Nugayev, R.M., Bashkov, V.I.: Particle creation of a black hole as a consequence of the Casimir effect. Phys. Lett. 69 A, 385 (1979)

3. Nugayev, R.M.: Particle creation by a black hole as a consequence of the Casimir effect. Phys. Lett. 91 A, 216 (1982) 
4. Nugayev, R.M.: Particle creation by a black hole as a consequence of quantum-field effects in flat space-time. Nuov. Cim. 86 B, 90 (1985)

5. Fabbri, R.: Scattering and absorption of electromagnetic waves by a Schwarzschild black hole. Phys. Rev. D 12, 933 (1975)

6. Casimir, H.B.G.: Proc. Kon. Ned. Akad. Wetenschap 51, 793 (1948)

7. Boyer, T.H.: Quantum zero-point energy and long-range forces. Ann. Phys. (N.Y.) 56, 474 (1970)

8. De Witt, B.S.: Quantum field theory in curved space-time. Phys. Rep. C 19, 295 (1975)

9. Boyer, T.H.: Quantum electromagnetic zero-point energy of a conducting spherical shell and the Casimir model for a charged particle. Phys. Rev. 174, 1764 (1968)

10. Davies, B.: Quantum electromagnetic zero-point energy of a conducting spherical shell. J. Math. Phys. 13, 1324 (1972)

11. Olaussen, K., Ravndal, F.: Chronomagnetic vacuum fields in a spherical bag. Phys. Lett. 100 B, 497 (1981); Electromagnetic vacuum fields in a spherical cavity, Nucl. Phys. B 192, 237 (1981)

12. Tadaki, S., Takagi, S.: Casimir effect at finite temperature. Progr. Theor. Phys. 75, 262 (1986)

13. Kennedy, L., Critchley, R., Dowker, J.S.: Finite temperature field theory with boundaries: Stress tensor and surface action renormalisation. Ann. Phys. (N.Y.) 125, 346 (1980)

14. Levin, M.L., Polevoy, V.G., Ritov, S.M.: Theory of heat transfer due to a fluctuation electromagnetic field. Zh. Eksp. Teor. Fiz. 79, 612 (1980)

15. Sanchez, N.: Absorption and emission spectra of a Schwarzschild black hole. Phys. Rev. D 18, 1030 (1978)

16. Sciama, D.W., Candelas, P., Deutsch, D.: Quantum field theory, horizons and thermodynamics. Adv. Phys. 30, 327 (1981)

17. Misner, C., Thorne, K., Wheeler, J.: Gravitation, San Francisco 1973

18. Bardeen, J.M., Carter, B., Hawking, S.W.: The four laws of black hole mechanics. Commun. Math. Phys. 31, 161 (1973)

19. Macdonald, D.A., Suen, W.M.: Membrane viewpoint on black holes: Dynamical electromagnetic fields near the horizon. Phys. Rev. D 32, 848 (1985)

20. Frolov, V.P., Zel'nikov, A.I.: Effect of vacuum polarization near black holes. In: Quantum gravity: Proceedings of the third seminar on quantum gravity, Moscow, 1984. Berezin, V.A. et al. (eds.). Singapore: World Sci. Publ. 1985

21. Page, D.N.: Thermal stress tensors in static Einstein spaces. Phys. Rev. D 25, 1499 (1982)

22. Gibbons, L.W., Perry, M.J. : Black holes and thermal Green functions. Proc. Roy. Soc. A 358, 467 (1978)

Communicated by L. Alvarez-Gaumé

Received October 29, 1986 